\section{(6) OPEN ACCESS}

\title{
Reducing the clinical burden of ranibizumab treatment for neovascular age-related macular degeneration using an individually planned regimen
}

\author{
Irmela Mantel, Sophie-Alexia Niderprim, Christina Gianniou, Angeliki Deli, \\ Aude Ambresin
}

- Additional material is published online only. To view please visit the journal online (http://dx.doi.org/10.1136/ bjophthalmol-2013-304556).

Department of Ophthalmology, University of Lausanne, Jules Gonin Eye Hospital, Lausanne, Switzerland

\section{Correspondence to} Dr Irmela Mantel, Department of Ophthalmology, University Eye Hospital Jules Gonin, 15 Av. de France, Case postale 133, CH-1000 Lausanne 7. Switzerland;

irmela.mantel@fa2.ch

Received 30 October 2013 Revised 19 February 2014 Accepted 20 March 2014 Published Online First 11 April 2014

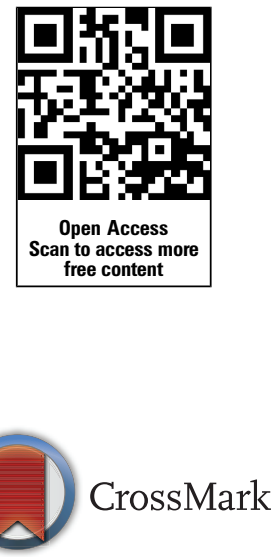

To cite: Mantel I, Niderprim S-A, Gianniou C, et al. Br J Ophthalmol 2014;98:1192-1196.

\section{ABSTRACT}

Aims The purpose of this study was to clinically validate an individually planned treatment regimen for neovascular age-related macular degeneration (nAMD), termed, observe and plan. This regimen was based on the predictability of an individual's need for retreatment and aimed to reduce the clinical burden, while obtaining good functional results.

Methods This was a prospective case series that included 104 patients (115 eyes) with treatment-naive nAMD. Following three loading doses of ranibizumab, monthly observation visits allowed the disease recurrence interval to be determined. The recurrence interval was reduced by 2 weeks to give the retreatment interval for the next three injections. Periodical control visits (at least every 6 months) allowed the effectiveness of the treatment to be assessed and individual intervals adjusted.

Results Mean visual acuity (VA) improved by 8.7 and 9.8 letters in months 3 and 12, respectively. The mean number of injections during the 12-month study was 7.8 , while the mean number of ophthalmic examinations between months 3 and 12 was 3.97. The mean treatment interval after the loading doses was 1.97 months.

Conclusions The observe-and-plan regimen significantly improved VA. This was obtained with fewer clinic visits compared with other regimens, which could ease the burden of nAMD treatment.

Trial registration number Commission cantonale (VD) d'éthique de la recherché Clinique, Université de Lausanne, Protocole 351/11.

\section{INTRODUCTION}

In 2002, advanced age-related macular degeneration (AMD) was the leading cause of irreversible vision loss in individuals aged 50 years and older in industrialised countries. ${ }^{1}$ Large multicentre, randomised controlled trials have shown that monthly intravitreal injections of ranibizumab, a specific recombinant, humanised $\operatorname{IgG}$ monoclonal antibody that neutralises all active forms of VEGF-A, ${ }^{2}$ significantly improve visual acuity (VA) in neovascular AMD (nAMD) compared with sham treatment, ${ }^{3}$ or photodynamic therapy. ${ }^{3-5}$ However, monthly injections place a heavy burden on the chronic care management of patients with nAMD. ${ }^{6}$

To alleviate this burden, more recent clinical trials have examined alternative treatment regimens. A regimen of injections every 3 months resulted in the loss of initial VA improvement, significantly inferior to monthly injections. ${ }^{7-9}$ An individualised dosing regimen, pro re nata (PRN), was investigated in the PrONTO, ${ }^{10}{ }^{11}$ CATT, ${ }^{12}{ }^{13}$ IVAN $^{14}$ and HARBOR ${ }^{15}$ clinical trials, during which it was found to be comparable to monthly dosing. Individualised dosing regimens are used frequently in clinical practice ${ }^{10} 11^{16-20}$ to reduce injection frequency while still obtaining good functional results compared with monthly retreatment. ${ }^{12} 1415$ However, with the exception of the treat and extend regimen (TER), ${ }^{17}$ these dosing schedules still require monthly monitoring to detect disease recurrences as early as possible.

We recently published the results from a study that evaluated the predictability of the need for retreatment across a group of 39 individuals with treatment-naive nAMD. ${ }^{21}$ We demonstrated the presence of a highly regular, predictable, individual pattern in the need for retreatment, with relatively small fluctuations over the course of 12 months. ${ }^{21}$ The present study aims to convert these results into a clinically relevant treatment protocol, using a planned treatment regimen based on the measurement of an individual's first disease recurrence interval. If successful, this novel treatment regimen will reduce the number of clinic visits and injections while still maintaining VA improvements throughout the follow-up period.

\section{METHODS}

This prospective study was undertaken in the medical retina department of a single tertiary referral centre (University Eye Hospital Jules Gonin in Lausanne, Switzerland). The study was approved by the local ethics committee (Commission d'éthique de la recherche clinique, Université de Lausanne) and adhered to the tenets of the Declaration of Helsinki. All patients gave written informed consent.

\section{Patient selection}

The study enrolled a consecutive series of patients (prospective aim was 100 patients) aged 50 years or older, with newly diagnosed, treatment-naive nAMD and active subfoveal choroidal neovascularisation (CNV), as confirmed on fluorescein angiography by a retinal specialist (AA or IM). Additional inclusion criteria were: best corrected VA (BCVA) from $20 / 25$ to $200 / 400$, a maximum lesion size of 12 disc areas, and informed consent. Exclusion criteria included: subfoveal atrophy or fibrosis, polypoidal choroidal vasculopathy, $\mathrm{CNV}$ resulting from a disease other than AMD, any other macular pathology, a history of any macular treatment or 
vitrectomy, aphakia, history of uveitis or active ocular inflammation in the study eye, any other vision-affecting ocular disorder, and the inability to obtain retinal images of sufficient quality. Therefore, the inclusion and exclusion criteria were similar to the multicentre phase III trials, ${ }^{3} 4{ }^{10}$ with the exception of a slightly enlarged spectrum for BCVA.

\section{Clinical investigations}

Baseline examination and all subsequent follow-up visits included measurement of BCVA on the Early Treatment of Diabetic Retinopathy Study (ETDRS) chart, slit lamp examination, measurement of intraocular pressure, dilated fundus examination, and spectral domain OCT (SD-OCT; Cirrus, Carl Zeiss Meditec, Oberkochen, Germany). The following additional examinations were performed at baseline and after 3 months, using the Topcon TRC-50IX (Tokyo, Japan): fundus autofluorescence imaging, fluorescein angiography, and indocyanine green angiography (the latter at baseline only).

\section{OCT analysis}

A macular cube $(512 \times 126)$ scan captured using an SD-OCT Cirrus machine was used for baseline examination and all follow-up visits. The macular thickness map was acquired with the integrated software 6.0.0.599, which centred on the automatically identified foveal pit and was manually corrected if needed. Central retinal thickness (CRT) was measured in the central $1 \mathrm{~mm}^{2}$ subfield. All OCT scans were qualitatively evaluated for the presence or absence of intraretinal or subretinal fluid.

\section{Observe-and-plan regimen}

The concept of the observe-and-plan regimen was to evaluate the individual need for retreatment in an observation phase after three loading doses, and then to apply the treatment in an individually adapted treatment plan of a series of injections with fixed intervals. The interval was regularly adjusted for the subsequent treatment plans. The details of the regimen (illustrated in figure 1) were as follows:

All patients underwent an initial loading dose of threemonthly intravitreal injections of $0.5 \mathrm{mg}$ ranibizumab (Lucentis, Novartis Pharma AG, Basel, Switzerland). The loading dose was followed by monthly observation visits (with extension allowed every 1.5 months and 2 months after 3 months and 6 months since the last injection, respectively), which allowed the determination of the interval from the third injection until first signs of exudative activity on SD-OCT or fundus examination. Based on this interval which was shortened by 2 weeks in an attempt to keep the macula dry, an individualised fixed treatment plan (without intermittent ophthalmic examination) was established for the next three injections. The available choice of intervals was limited to 1-3 months. Longer intervals were not allowed because of unavailable data about their reliability. ${ }^{21}$ Therefore, recurrences that were observed at 4 months or more since last injection, were an indication to treat every 3 months. A recurrence after 2 months and 3 months was an indication for retreatment every 1.5 and 2.5 months, respectively, while eyes with exudative signs already at 1 month after last injection continued on the monthly retreatment regimen for the next three injections. Because of the concern of undiagnosed intermittent recurrence once the individualised fixed treatment plan has been launched, we decided to see the patient at least every 6 months for ophthalmic examination. Therefore, only two injections were planned for intervals of 2.5 or 3 months, with an assessment visit after 5 months and 6 months, respectively.

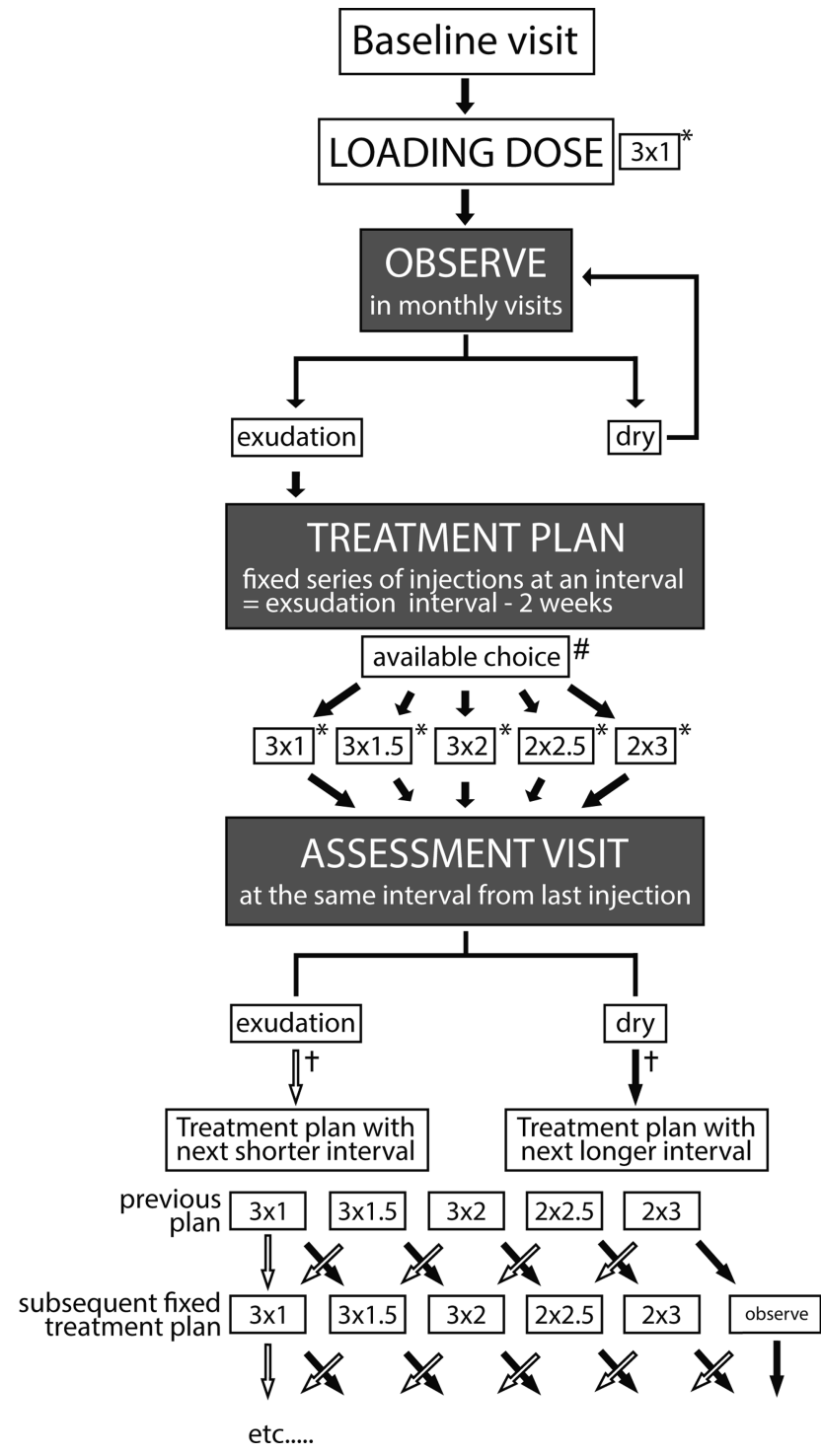

Figure 1 Treatment algorithm of this observe-and-plan regimen with ranibizumab for neovascular age-related macular degeneration. Every patient received the initial loading dose of 3 ranibizumab injections at 1 -month intervals, (abbreviated as $3 \times 1$ ). Subsequently, findings on optical coherence tomography defined the pathway in the algorithm; $3 \times 1^{*}=$ three injections at fixed interval of 1 month and assessment visit 1 month after the third injection (total assessment interval 3 months); $3 \times 1.5^{*}=$ three injections at fixed interval of 1.5 months and assessment visit 1.5 months after the third injection (total assessment interval 4.5 months); $3 \times 2{ }^{*}=$ three injections at fixed interval of 2 months and assessment visit 2 months after the third injection (total assessment interval 6 months); $2 \times 2.5^{*}=$ two injections at fixed interval of 2.5 months and assessment visit 2.5 months after the third injection (total assessment interval 5 months); $2 \times 3^{*}=$ two injections at fixed interval of 3 months and assessment visit 3 months after the third injection (total assessment interval 5 months). "The possible treatment intervals for the fixed injection plan ranged from a minimum of 1 month to a maximum of 3 months. In case of an injectionrecurrence interval more than 3 months - the patient received the treatment plan of two injections every 3 months $\left(2 \times 3\right.$ months). ${ }^{\dagger}$ Empty arrow=decision in case of exudative signs on ophthalmic examination, full arrow=decision in case of dry macula on ophthalmic examination.

Figure 1 gives an overview over the available choice of fixed treatment series (3 times 1, 1.5, or 2 months; twice 2.5 or 3 months). 
Follow-up assessment visits were performed with the same interval from the last injection of the series, and allowed for a dynamic feedback mechanism and adjustment of the intervals in the subsequent treatment plans. In the presence of exudative signs, the future treatment interval was shortened by 2 weeks (to a minimum of 1 month). In the absence of exudative signs, the future treatment interval was extended by 2 weeks (to a maximum of 3 months). However, if the treatment interval was successfully lengthened to 3 months, and the macula remained dry, the patient was given the option of continuing to receive a series of two injections every 3 months, or being monitored every 1.5 months. In case of intermittent or severe exudative recurrence, the protocol allowed shortening of the interval at the investigator's discretion.

\section{Clinical outcome analysis}

Clinical outcome measures included the following: mean BCVA change over time with an endpoint at 12 months; the proportion of eyes that lost $<15$ letters; the proportion of eyes that gained $\geq 15$ letters; mean CRT change; the initial interval after the loading dose; the mean treatment interval during the maintenance phase; the number of visits and injections over 12 months. The last available BCVA and CRT measurements were carried forward to the last visit to allow for statistical analysis. However, missing data for patients who were lost to follow-up were taken into account until their last visit only. These patients were not included in the analysis of stability versus changing need for retreatment over time. An economic assessment of this regimen in comparison with other variable treatment protocols was completed (see online supplementary methods).

Statistical results were considered significant at a level of significance of 0.05 .

\section{RESULTS}

\section{Baseline characteristics}

A total of 115 eyes from 104 patients were included in this study (107 eligible patients; 3 patients did not give informed consent); 102 patients (113 eyes) completed 12 months follow-up (1 patient was lost after 10 months for departure to another country, 1 patient changed hospitals after 6 months). The mean age was 79.5 years (SD 7.8, range 59-95; table 1) and included 66 women (63.5\%). All patients were Caucasian.

\section{Improvements in VA and CRT}

Mean BCVA improved significantly from 58.3 to 67.0 ETDRS letters $(+8.7$ compared with baseline; $\mathrm{p}<0.0001$, paired $t$ test $)$ at month 3 , and this increase was maintained to month 12 (+9.8 letters) (figure 2; upper graph). By month 12, $98 \%$ of eyes had lost less than 15 ETDRS letters (3 lines), 84\% had gained $\geq 0$ letters, and $30 \%$ had gained $\geq 15$ ETDRS letters. Loss of more than 15 letters ETDRS was related to increasing atrophy. OCT measurements demonstrated that mean CRT improved from $342 \mu \mathrm{m}$ (SD 85, range 186-689) at baseline by a mean of -97 and $-99 \mu \mathrm{m}$ at months 3 and 12 , respectively (figure 2; lower graph).

\section{Factors impacting upon clinical burden}

The mean number of injections performed during the first 12 months was 7.8 (SD 3.1, range 3-13), including the first three loading doses. Between months 3 and 12, the mean number of clinic visits, with ophthalmic examinations, was 3.97 (SD 1.40, range 2-8; figure 3) and the mean treatment interval was 1.97 months. After the loading doses, 35\% of eyes showed exudative signs 1 month after the third injection. Half these eyes (17\%) did not show absence of subretinal and intraretinal fluid on SD-OCT until month 12, and therefore remained on this monthly dosing regimen. The observation phase resulted in a $1.5,2$ to 2.5 , and 3 months interval in the first retreatment plan for $19 \%, 16 \%$ and $18 \%$ of eyes, respectively. The macula remained exudation-free until month 12 in $12 \%$ of eyes.

An explorative analysis of potentially interval-associated factors (age, lesion size, lesion type, baseline CRT, baseline VA) remained negative. However, the numbers included are insufficient for this analysis. Interestingly, 8 out of the 11 patients with bilateral eye inclusion (72.7\%) showed highly symmetric need for retreatment with a maximum of one injection difference in the first year. This might suggest that systemic factors, rather than ocular factors, play a more important role for the need of retreatment.

Within the follow-up period, the treatment interval remained stable (ie, within \pm 2 weeks) in $80 \%$ of eyes. The interval was progressively lengthened in $15 \%$ of eyes, while $5 \%$ of eyes needed a shortening of the interval by more than 2 weeks as compared to the first measured interval. The direct medical cost of this observe-and-plan regimen was near-equivalent to the variable dosing arm of the CATT protocol (see online supplementary table S1).

Table 1 Comparison of baseline characteristics between different ranibizumab treatment regimens for nAMD

\begin{tabular}{|c|c|c|c|c|c|}
\hline & MARINA $^{3}$ & ANCHOR $^{4}$ & $\mathrm{CATT}^{12}$ & $\mathrm{TER}^{17}$ & Observe and plan* \\
\hline Gender (\% female) & 63.3 & 46.4 & 61.9 & 66.3 & 63.5 \\
\hline Race (\% Caucasian) & 96.7 & 97.1 & 98.6 & 100 & 100 \\
\hline Age $($ mean $\pm S D)$ & $77 \pm 8.0$ & $76 \pm 8.6$ & $79.3 \pm 7.8$ & $80.6 \pm 6.6$ & $79.5 \pm 7.8$ \\
\hline VA mean (median) & na & na & $20 / 60^{+1}$ & $20 / 135(20 / 100)$ & $20 / 80^{+3}\left(20 / 60^{+3}\right)$ \\
\hline$V A \leq 20 / 200(\%)$ & 12.9 & 23.0 & 6.8 & 22.8 & 11.3 \\
\hline $20 / 200<\mathrm{VA}<20 / 40$ & 72.1 & 72.7 & 62.3 & 67.4 & 58.3 \\
\hline$V A \geq 20 / 40$ & 15.0 & 4.3 & 30.9 & 9.8 & 30.4 \\
\hline Predominately classic CNV lesion (\%) & 0 & 96.4 & na & 19.6 & 24.4 \\
\hline Minimally classic CNV lesion (\%) & 37.9 & 3.6 & na & 28.7 & 10.4 \\
\hline Occult with no classic CNV (\%) & 62.1 & 0 & na & 52.5 & 45.2 \\
\hline Retinal angiomatous proliferation (\%) & na & na & na & na & 20.0 \\
\hline Size of lesion (disc areas) & 4.5 & 1.79 & 2.7 & 2.6 & 2.48 \\
\hline
\end{tabular}

*Treatment regimen of this study.

CNV, choroidal neovascularisation; na, not applicable; nAMD; neovascular age-related macular degeneration; TER, treat and extend regimen; VA, visual acuity. 
Figure 2 Mean change of best corrected visual acuity (BCVA; upper graph) and of central retinal thickness (CRT) measured using optical coherence tomography (lower graph) of all study eyes treated with ranibizumab for neovascular age-related macular degeneration following an observe and plan regimen during the 12 month study period. Error bars represent SE of the mean.

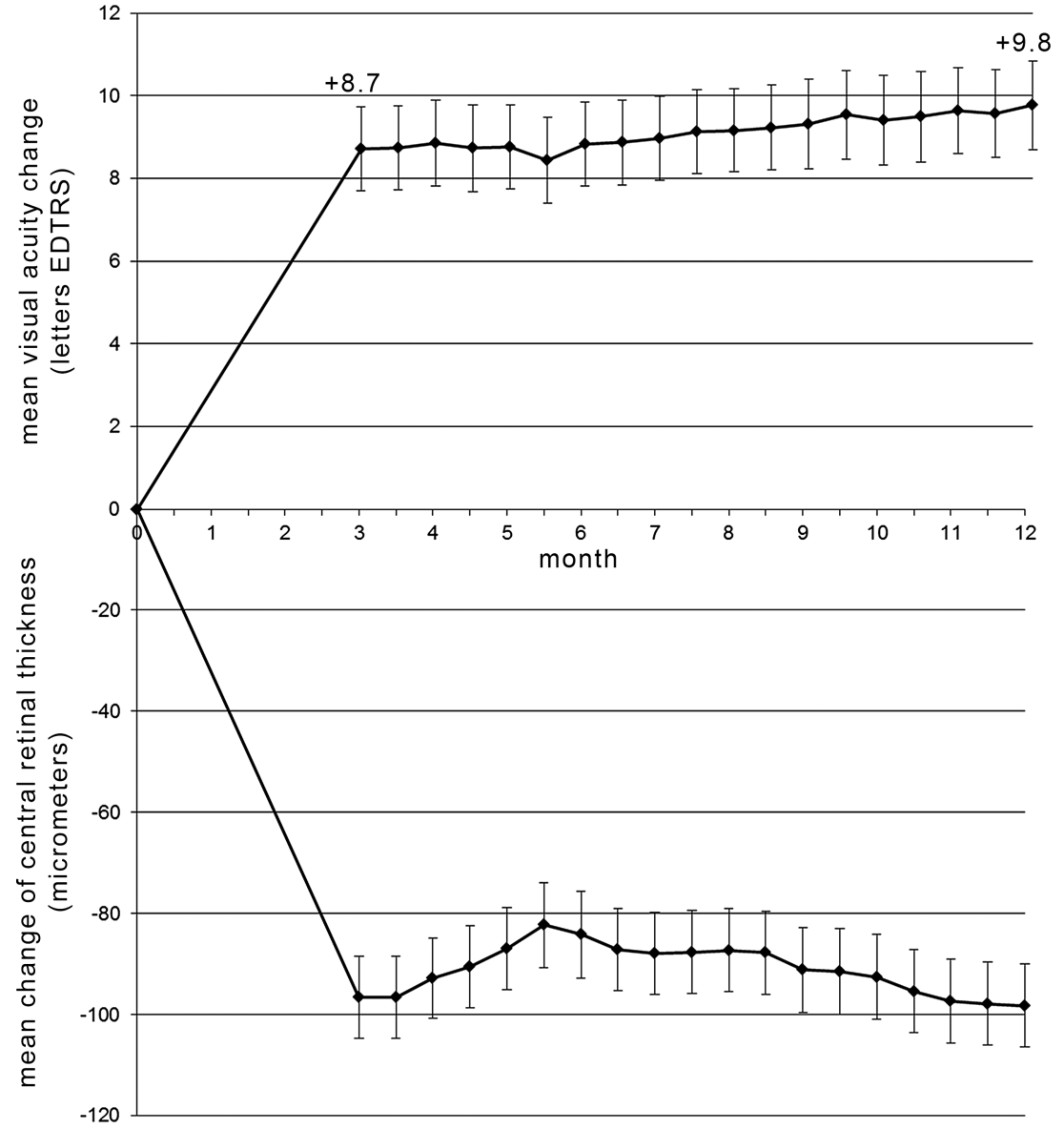

No severe ocular or systemic adverse events were reported during the course of this study.

\section{DISCUSSION}

In the present study, we demonstrated the success of an individually tailored observe-and-plan regimen, which dramatically reduced the number of clinic visits with ophthalmic examinations, yet obtained good functional outcomes over 12 months through the administration of the appropriate number of injections. These results are important in two ways.

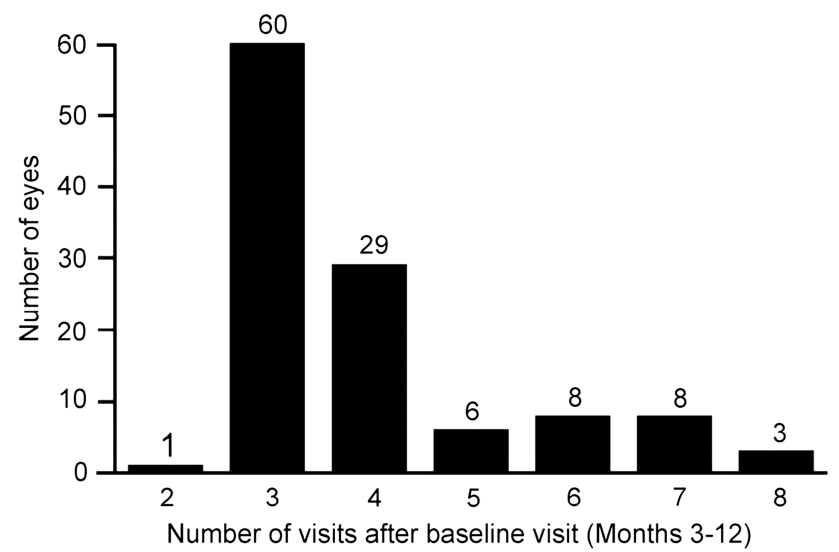

Figure 3 Distribution of the number of clinic visits with ophthalmic examination between months 3 and 12 .
First, the large reduction of clinic visits that require detailed ophthalmologic examinations, to a mean of just below four in the first year of the study, should allow clinical teams to manage the treatment burden of nAMD more efficiently, compared with the 12 visits required by PRN regimens ${ }^{12} 1415$ or the $\sim 8$ visits involved for TER. ${ }^{17}$ In fact, these examination visits are the time-consuming part of patient care, and their number determines the clinical burden. Clinical burden is an important, but often ignored, factor in determining the optimal treatment regimen to use, and unnecessary examinations waste clinical resources that could be better applied elsewhere. ${ }^{6}$ With observe-and-plan regimens, two to three more patients per day may be managed in clinics as compared with PRN and TER treatment routines. However, the number of injections remained similar within these three regimens (observe-and-plan, PRN and TER), as observe-and-plan was designed to anticipate each individual's requirement for injections.

Second, the results of this study support that individualised predicting future treatment needs for up to 6 months, on the basis of the measurement of the first recurrence interval, combined with a dynamic feedback evaluation loop (ie, assessment visits; figure 1), provides adequate treatment for nAMD. The final visual outcome and, particularly, the maintenance of the initial visual gain (figure 2), served as validation of this regimen. It has been repeatedly observed that late retreatment ${ }^{8} 9$ and $\mathrm{PRN}^{9} 18 \quad 22 \quad 23$ are associated with secondary loss of the initial visual improvement to various degrees. While the initial improvement in VA at month 3-after the three loading doses-reflects the potential of the photoreceptors to recover function, the most important phase for the validation of any retreatment regimen is 
the maintenance phase after month 3. Thus far, various studies have concluded that strict monthly visits are mandatory in a PRN regimen to maintain the initial VA improvement past month $3 .^{10} 1123$ By contrast, our results that show slight improvement between month 3 and month 12 (figure 2), suggest that individually planned injection schedules using the predictability of need for retreatment may allow similarly good outcomes, even though far fewer examination visits are required.

Clinicians may be concerned about a possible risk of undertreatment of patients with nAMD, due to the departure from monthly ophthalmic examinations. Data presented herein argue against this concern in three ways. First, throughout the follow-up assessment visits, no patient presented with a strong exudative recurrence, which indicated that the planned intervals were appropriately tailored to treat nAMD in each individual. Second, only $2 \%$ of eyes lost more than three lines of BCVA after 12 months, which is comparable with the MARINA and ANCHOR trials, where this number was about $5 \% .^{3} 4$ The third observation was that only $5 \%$ of patients actually required a reduction of their retreatment interval compared with that initially estimated for them. Observe-and-plan, therefore, did not lead to the undertreatment of patients in this study.

In conjunction with the benefits of the regimen, there was one potential limitation. The mean number of reinjections in the first year was slightly higher than in the PRN arms of the multicentre studies (7.8 instead of 6.9-7.7), ${ }^{12} 1315$ possibly due to the fixed dosing phases, and partially due to sensitive retreatment criteria used in this study (SD-OCT was only used in the HARBOR trial).

We acknowledge that the present study was uncontrolled. The regimen was validated only in that it maintained the VA gain after month 3 through to month 12 .

In conclusion, chronic care management of patients with nAMD, using the observe-and-plan regimen, based on the predictability of the need for retreatment, resulted in significant and maintained VA improvement. The gains in VA were achieved with a number of injections comparable with other variable dosing regimens (PRN or TER), but required fewer clinic visits with ophthalmic examinations. This variable dosing regimen has the potential to ease the burden of nAMD treatment management on institutions and doctors, and may help with patient compliance. However, long-term data beyond a 12-month follow-up period, and direct comparison with the gold standard of monthly retreatment, are needed in order to definitively confirm its value.

Correction notice This article has been corrected since it was published Online First. In the Results section of the Abstract, the value 2.08 has been changed to 1.97 in the sentence 'The mean treatment interval after the loading doses was 1.97 months.'

Acknowledgements The authors acknowledge the assistance of Fishawack Communications Ltd in editing this manuscript.

Contributors Design of the study: IM and AA. Conduct of the study: AD, CG, AA and IM. Collection and management of the data: CG, S-AN, AD and IM. Analysis and interpretation of the data: IM. Preparation of the manuscript: S-AN and IM. Review and approval of the manuscript: IM, AA, CG, AD and S-AN.

Funding Medical writing support was provided by Gillian Groeger, Fishawack Communications Ltd, UK, and was funded by Novartis.

Competing interests IM declared medical writing support funded by Novartis for the development of the manuscript.

Ethics approval Commission d'éthique de la recherche clinique, Université de Lausanne.

Provenance and peer review Not commissioned; externally peer reviewed.
Open Access This is an Open Access article distributed in accordance with the Creative Commons Attribution Non Commercial (CC BY-NC 3.0) license, which permits others to distribute, remix, adapt, build upon this work non-commercially, and license their derivative works on different terms, provided the original work is properly cited and the use is non-commercial. See: http://creativecommons.org/ licenses/by-nc/3.0/

\section{REFERENCES}

1 Resnikoff S, Pascolini D, Etya'ale D, et al. Global data on visual impairment in the year 2002. Bull World Health Organ 2004;82:844-51.

2 Ferrara N, Damico L, Shams N, et al. Development of ranibizumab, an anti-vascular endothelial growth factor antigen binding fragment, as therapy for neovascular age-related macular degeneration. Retina 2006;26:859-70.

3 Rosenfeld PJ, Brown DM, Heier JS, et al. Ranibizumab for neovascular age-related macular degeneration. N Engl J Med 2006;355:1419-31.

4 Brown DM, Kaiser PK, Michels $M$, et al. Ranibizumab versus verteporfin for neovascular age-related macular degeneration. N Engl J Med 2006;355:1432-44.

5 Brown DM, Michels M, Kaiser PK, et al. Ranibizumab versus verteporfin photodynamic therapy for neovascular age-related macular degeneration: Two-year results of the ANCHOR study. Ophthalmology 2009:116:57-65.

6 Han DP. Age-related macular degeneration, anti-VEGF therapy, and ophthalmic imaging: is there a best practice? JAMA Ophthalmol 2013;131:1124-6.

7 Abraham P, Yue H, Wilson L. Randomized, double-masked, sham-controlled trial of ranibizumab for neovascular age-related macular degeneration: PIER study year 2 . Am J Ophthalmol 2010;150:315-24.

8 Regillo CD, Brown DM, Abraham P, et al. Randomized, double-masked, sham-controlled trial of ranibizumab for neovascular age-related macular degeneration: PIER Study year 1. Am J Ophthalmol 2008;145:239-48.

9 Schmidt-Erfurth U, Eldem B, Guymer R, et al. Efficacy and safety of monthly versus quarterly ranibizumab treatment in neovascular age-related macular degeneration: the EXCITE study. Ophthalmology 2011;118:831-9.

10 Fung AE, Lalwani GA, Rosenfeld PJ, et al. An optical coherence tomography-guided, variable dosing regimen with intravitreal ranibizumab (Lucentis) for neovascular age-related macular degeneration. Am J Ophthalmol 2007;143:566-83.

11 Lalwani $G A$, Rosenfeld PJ, Fung $A E$, et al. A variable-dosing regimen with intravitreal ranibizumab for neovascular age-related macular degeneration: year 2 of the PrONTO Study. Am J Ophthalmol 2009;148:43-58.

12 Martin DF, Maguire MG, Fine SL, et al. Ranibizumab and bevacizumab for treatment of neovascular age-related macular degeneration: two-year results. Ophthalmology 2012;119:1388-98.

13 Martin DF, Maguire MG, Ying GS, et al. Ranibizumab and bevacizumab for neovascular age-related macular degeneration. N Engl J Med 2011;364:1897-908.

14 Chakravarthy U, Harding SP, Rogers CA, et al. Alternative treatments to inhibit VEGF in age-related choroidal neovascularisation: 2-year findings of the IVAN randomised controlled trial. Lancet 2013:382:1258-67.

15 Busbee BG, Ho AC, Brown DM, et al. Twelve-month efficacy and safety of $0.5 \mathrm{mg}$ or $2.0 \mathrm{mg}$ ranibizumab in patients with subfoveal neovascular age-related macular degeneration. Ophthalmology 2013;120:1046-56.

16 Cohen SY, Dubois L, Tadayoni $R$, et al. Results of one-year's treatment with ranibizumab for exudative age-related macular degeneration in a clinical setting. Am J Ophthalmol 2009:148:409-13.

17 Gupta OP, Shienbaum G, Patel AH, et al. A treat and extend regimen using ranibizumab for neovascular age-related macular degeneration clinical and economic impact. Ophthalmology 2010;117:2134-40.

18 Dadgostar H, Ventura AA, Chung JY, et al. Evaluation of injection frequency and visual acuity outcomes for ranibizumab monotherapy in exudative age-related macular degeneration. Ophthalmology 2009;116:1740-7.

19 Mantel I, Zografos L, Ambresin A. Early clinical experience with ranibizumab for occult and minimally classic neovascular membranes in age-related macular degeneration. Ophthalmologica 2008:222:321-3.

20 Oubraham H, Cohen SY, Samimi S, et al. Inject and extend dosing versus dosing as needed: a comparative retrospective study of ranibizumab in exudative age-related macular degeneration. Retina 2011;31:26-30.

21 Mantel I, Deli A, Iglesias K, et al. Prospective study evaluating the predictability of need for retreatment with intravitreal ranibizumab for age-related macular degeneration. Graefes Arch Clin Exp Ophthalmol 2013;251:697-704.

22 Boyer DS, Heier JS, Brown DM, et al. A phase Illb study to evaluate the safety of ranibizumab in subjects with neovascular age-related macular degeneration. Ophthalmology 2009:116:1731-9.

23 Holz FG, Amoaku W, Donate J, et al. Safety and efficacy of a flexible dosing regimen of ranibizumab in neovascular age-related macular degeneration: the SUSTAIN study. Ophthalmology 2011;118:663-71. 